\title{
Prognostic Value of Pretherapeutic Tumor-to-Blood Standardized Uptake Ratio in Patients with Esophageal Carcinoma
}

\author{
Rebecca Bütof*1,2, Frank Hofheinz*3, Klaus Zöphel ${ }^{2,4,5}$, Tobias Stadelmann ${ }^{4}$, Julia Schmollack ${ }^{4}$, Christina Jentsch ${ }^{1,2}$, \\ Steffen Löck ${ }^{2}$, Jörg Kotzerke ${ }^{2,4,5}$, Michael Baumann ${ }^{1,2,5,6}$, and Jörg van den Hoff ${ }^{3,4}$ \\ ${ }^{I}$ Department of Radiation Oncology, University Hospital Carl Gustav Carus, Technische Universität Dresden, Dresden, Germany; \\ ${ }^{2}$ OncoRay-National Center for Radiation Research in Oncology, Faculty of Medicine and University Hospital Carl Gustav Carus, \\ Technische Universität Dresden, Helmholtz-Zentrum Dresden-Rossendorf, Dresden, Germany; ${ }^{3}$ Helmholtz-Zentrum Dresden- \\ Rossendorf, PET Center, Institute of Radiopharmaceutical Cancer Research, Dresden, Germany; ${ }^{4}$ Department of Nuclear Medicine, \\ University Hospital Carl Gustav Carus, Technische Universität Dresden, Dresden, Germany; ${ }^{5}$ German Cancer Consortium (DKTK), \\ Dresden and German Cancer Research Center (DKFZ), Heidelberg, Germany; and ${ }^{6}$ Helmholtz-Zentrum Dresden-Rossendorf, \\ Institute of Radiooncology, Dresden, Germany
}

Despite ongoing efforts to develop new treatment options, the prognosis for patients with inoperable esophageal carcinoma is still poor and the reliability of individual therapy outcome prediction based on clinical parameters is not convincing. The aim of this work was to investigate whether PET can provide independent prognostic information in such a patient group and whether the tumor-toblood standardized uptake ratio (SUR) can improve the prognostic value of tracer uptake values. Methods: ${ }^{18} \mathrm{~F}-\mathrm{FDG}$ PET/CT was performed in 130 consecutive patients (mean age \pm SD, $63 \pm 11$ y; 113 men, 17 women) with newly diagnosed esophageal cancer before definitive radiochemotherapy. In the PET images, the metabolically active tumor volume (MTV) of the primary tumor was delineated with an adaptive threshold method. The blood standardized uptake value (SUV) was determined by manually delineating the aorta in the low-dose CT. SUR values were computed as the ratio of tumor SUV and blood SUV. Uptake values were scan-time-corrected to 60 min after injection. Univariate Cox regression and Kaplan-Meier analysis with respect to overall survival (OS), distant metastases-free survival (DM), and locoregional tumor control (LRC) was performed. Additionally, a multivariate Cox regression including clinically relevant parameters was performed. Results: In multivariate Cox regression with respect to OS, including $\mathrm{T}$ stage, $\mathrm{N}$ stage, and smoking state, MTV- and SUR-based parameters were significant prognostic factors for OS with similar effect size. Multivariate analysis with respect to DM revealed smoking state, MTV, and all SUR-based parameters as significant prognostic factors. The highest hazard ratios (HRs) were found for scan-time-corrected maximum SUR $(H R=3.9)$ and mean SUR $(H R=4.4)$. None of the PET parameters was associated with $\mathrm{LRC}$. Univariate Cox regression with respect to $L R C$ revealed a significant effect only for $\mathrm{N}$ stage greater than $0(P=0.048)$. Conclusion: PET provides independent prognostic information for OS and DM but not for LRC in patients with locally advanced esophageal carcinoma treated with definitive radiochemotherapy in addition to clinical parameters. Among the investigated uptake-based parameters, only SUR

Received Feb. 4, 2015; revision accepted Jun. 6, 2015

For correspondence or reprints contact: Rebecca Bütof, Department of Radiation Oncology, University Hospital Carl Gustav Carus, Fetscherstrasse 74, 01307 Dresden, Germany.

E-mail: Rebecca.Buetof@uniklinikum-dresden.de

${ }^{*}$ Contributed equally to this work.

Published online Jun. 18, 2015.

COPYRIGHT (C) 2015 by the Society of Nuclear Medicine and Molecular Imaging, Inc. was an independent prognostic factor for OS and DM. These results suggest that the prognostic value of tracer uptake can be improved when characterized by SUR instead of SUV. Further investigations are required to confirm these preliminary results.

Key Words: PET; esophageal cancer; definitive radiochemotherapy; SUV; SUR

J Nucl Med 2015; 56:1150-1156

DOI: 10.2967/jnumed.115.155309

D espite ongoing efforts to develop new treatment options, esophageal cancer remains one of the world's most lethal malignancies. In early-stage esophageal carcinoma, surgical resection is still the mainstay of therapy. For locally advanced disease, several randomized trials have shown the benefit of neoadjuvant chemoradiation followed by surgery, compared with surgery alone, for example (1). In the case of inoperability due to comorbidities or functional reasons, definitive radiochemotherapy is applied. Unfortunately, prognosis for these patients is still poor, with an average overall survival (OS) of $15 \%$ after 5 y (2), and the reliability of individual therapy outcome prediction based on clinical parameters alone cannot be considered satisfactory. It would, therefore, be highly desirable to improve prediction of therapy outcome. One promising route is to combine optimized quantitative assessment of the additional functional information provided by ${ }^{18}$ F-FDG PET with proven clinical parameters. Ultimately, this may serve to advance individualized treatment schedules, for example, use of increased radiation dose in patients with a high risk of local recurrence or intensified chemotherapy schedules in the case of high risk of distant metastases.

The possible added value of ${ }^{18} \mathrm{~F}$-FDG PET for therapy assessment is well recognized. In fact, it has been demonstrated in several studies that pretherapeutic ${ }^{18} \mathrm{~F}$-FDG PET has the potential to provide prognostic information in addition to clinical parameters (e.g., histology, grading, $\mathrm{T}$ stage and $\mathrm{N}$ stage, age) in patients with esophageal carcinoma $(3,4)$. In most of these studies, the investigated patient group was treated with neoadjuvant chemoradiation followed by surgery or with surgery alone. Much less has been published regarding patients 
with inoperable tumors who are thus treated with definitive chemoradiation. We found only 4 publications in which baseline PET parameters were correlated with survival data in such a patient group. Suzuki et al. (5) and Atsumi et al. (6) showed that the maximum standardized uptake value $\left(\mathrm{SUV}_{\max }\right)$ was a prognostic factor for OS and relapse-free survival. On the other hand, in the investigation of

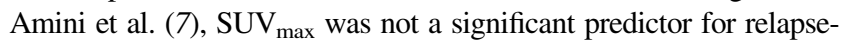
free survival. Lemarignier et al. (8) analyzed the prognostic value of several baseline PET parameters: $\mathrm{SUV}_{\text {max }}$, mean SUV ( $\mathrm{SUV}_{\text {mean }}$ ), the metabolically active tumor volume (MTV), and the product of MTV and $\mathrm{SUV}_{\text {mean }}$ (total lesion glycolysis [TLG]), respectively. In this investigation, $\mathrm{SUV}_{\max }$ was a prognostic factor for disease-free survival, but standardized uptake value (SUV) was inferior to MTV and was not predictive for OS.

An explanation for this unsatisfactory performance of SUV may be the adverse effects of well-known shortcomings of the SUV methodology - especially in the clinical setting - such as scan-time dependence of the SUV, interstudy variability of the arterial input function (AIF), susceptibility to errors in scanner calibration, and the like (9-12), all of which adversely affect the reliability of the SUV as a surrogate of the metabolic rate of glucose consumption, which in turn can be expected to reduce the prognostic value of SUV. Indeed, in a recent publication we demonstrated that the standard tumor-toblood SUV ratio (SUR) was superior to the (tumor) SUV itself as a surrogate parameter of metabolic rate of glucose consumption (13) for well-standardized uptake periods (i.e., scan start times). In the clinical context, variability of the uptake period is unavoidable, which directly translates into a corresponding variability of the measured tracer uptake. But as has been shown recently, it is possible to reliably correct SUR (and somewhat less reliably SUV) for variations of the ${ }^{18} \mathrm{~F}$-FDG uptake period (14) by converting the measured uptake values to a preselected fixed scan time point. This scan-timenormalized SUR removes several of the shortcomings of SUV, leading to a much improved linear correlation between this uptake parameter and the actually targeted quantity, namely the metabolic rate of ${ }^{18} \mathrm{~F}-\mathrm{FDG}$. However, so far it has not been investigated whether these principal advantages of SUR translate into an improved prognostic value of this parameter in comparison to SUV.

Therefore, the aim of this exploratory study was to evaluate the prognostic value of pretherapeutic SUR (with and without scantime correction) and scan-time-corrected SUV in comparison to the conventional parameters MTV, TLG, and SUV and also considering known clinical prognostic parameters in patients with esophageal carcinoma and a definitive chemoradiation treatment regime.

This investigation specifically tested the hypothesis that assessment of the pretherapeutic glucose metabolic rate (as measured by the lesion's SUR) is prognostic for therapy outcome in esophageal carcinoma. By comparing the prognostic value of SUR and SUV, the study also tested the hypothesis that the more accurate assessment of the lesion's metabolic rate by SUR instead of SUV translated into an improved value of the former parameter in comparison to SUV.

\section{MATERIALS AND METHODS}

\section{Patient Characteristics}

In the present study, 130 consecutive patients with ${ }^{18} \mathrm{~F}-\mathrm{FDG}$ PET/ CT-staged esophageal carcinoma were included retrospectively. Evaluation of the data was approved by the Institutional Ethics Committee, and all subjects signed a written informed consent form. All cases were discussed in an interdisciplinary tumor board, and patients-because of either locally advanced disease or comorbidities/functional inoperability- received curative definitive radiochemotherapy between September 2005 and October 2013. Inclusion criteria were age more than $18 \mathrm{y}$, histologically confirmed esophageal carcinoma, ${ }^{18} \mathrm{~F}-\mathrm{FDG}$ PET/CT staging, no distant metastases, curative treatment intention, and a minimum follow-up of $12 \mathrm{mo}$. All patients had a clinical staging of Union for International Cancer Control (UICC) stage based on PET imaging and endoscopy/endosonography. The median age of all included patients was $65 \mathrm{y}$ (range, 42-85 y), and most were male (87\%). A summary of patient and tumor characteristics is given in Table 1.

\section{Treatment}

All patients were treated with 3-dimensional CT-planned conformal radiotherapy. Total radiation doses of 60, 66, or 70 Gy (cervical tumor localization) were applied in 2 Gy per fraction. The gross tumor volume was separately delineated in the PET and CT images. Up to 50 Gy an elective nodal irradiation of the mediastinum was additionally performed. For boost irradiation, margins of $1.5 \mathrm{~cm}$ in axial and 3.5 $\mathrm{cm}$ in craniocaudal directions were used to create the clinical target volume. The chemotherapy schedule consisted of cisplatin $\left(70 \mathrm{mg} / \mathrm{m}^{2}\right)$ and 5 -fluorouracil $\left(3,000 \mathrm{mg} / \mathrm{m}^{2}\right.$ as an infusion over $\left.96 \mathrm{~h}\right)$ in week 1 and 4 of the treatment.

TABLE 1

Patient and Tumor Characteristics

\begin{tabular}{|cc}
\hline \multicolumn{1}{|c}{ Characteristic } & Value \\
\hline Age $(\mathrm{y})$ & \\
\hline Mean \pm SD & $63 \pm 11$ \\
\hline Median & 65 \\
\hline Sex & $113(87)$ \\
\hline Male & $17(13)$ \\
\hline Female & \\
\hline Histology & $106(82)$ \\
\hline Squamous cell carcinoma & $21(16)$ \\
\hline Adenocarcinoma & $3(2)$ \\
\hline Other & \\
\hline T stage & $7(5)$ \\
\hline T1 & $23(18)$ \\
\hline T2 & $82(63)$ \\
\hline T3 & $13(10)$ \\
\hline T4 & $5(4)$ \\
\hline Tx & \\
\hline N stage & \\
\hline N0 & \\
\hline N1 & $15(11)$ \\
\hline N2 & $35(27)$ \\
\hline N3 & $74(62)$ \\
\hline Nx & $7(57)$ \\
\hline I & $2(1)$ \\
\hline III stage & $6(5)$ \\
\hline
\end{tabular}

Except for patient age, all data entries are specified as number of occurrence, followed in parentheses by corresponding percentage fraction. 


\section{${ }^{18}$ F-FDG PET/CT Protocol}

All patients underwent a hybrid ${ }^{18} \mathrm{~F}-\mathrm{FDG}$ PET/CT scan before treatment. Scans (3-dimensional PET acquisition, 3-min emission per bed position) were obtained with a Biograph 16 (Siemens Medical Solutions Inc.). Data acquisition started $77 \pm 16$ min after injection of 212-417 MBq of ${ }^{18}$ F-FDG. All patients had fasted for at least $6 \mathrm{~h}$ before ${ }^{18} \mathrm{~F}$-FDG injection. The serum glucose concentration measured before injection was $5.8 \mu \mathrm{mol} / \mathrm{mL}$ on average (range, $3.3-9.8 \mu \mathrm{mol} / \mathrm{mL}$ ). Tomographic images were reconstructed using attenuationweighted ordered-subset expectation maximization reconstruction (4 iterations, 8 subsets, gaussian filter of $5 \mathrm{~mm}$ in full width at half maximum). The resulting image data had a voxel size of $4.1 \times 4.1 \times$ $5 \mathrm{~mm}$.

\section{Data Analysis}

Region-of-interest (ROI) definition and ROI analyses were performed using ROVER software (version 2.1.20; ABX). Here and in the following sections, ROI is used synonymously for VOI, for denoting a 3-dimensional volume of interest.

The metabolically active part of the primary tumor was delineated in the PET data by an automatic algorithm based on adaptive thresholding considering the local background (15). The resulting delineation was inspected visually by 2 experienced observers in consensus. Lesions were delineated manually where this was deemed necessary (observers were masked to patient outcome). Manual delineation happened in 5 of 130 cases exhibiting only low diffuse tracer accumulation in the respective lesion. In 6 further cases, the primary tumor was not visible in the PET data, and a small ROI $(<1 \mathrm{~mL})$ was manually placed in the esophagus.

For the delineated ROIs, SUV ${ }_{\text {max }}, \mathrm{SUV}_{\text {mean }}$, MTV, and TLG (MTV $\times$ $\mathrm{SUV}_{\text {mean }}$ ) were computed. The arterial blood SUV needed for computation of SUR values was determined by defining a roughly cylindric aorta ROI in the attenuation CT data, which then was transferred to the PET data (Fig. 1). To exclude partial-volume effects in this evaluation, a concentric safety margin was used in the transaxial planes, centering the ROI in the aorta. Planes showing high tracer uptake close to the aorta (pathologic or otherwise) were excluded. The minimum volume of the resulting aorta ROI was $5 \mathrm{~mL}$. Blood SUV was computed as the $\mathrm{SUV}_{\text {mean }}$ in the aorta ROI. Figure 1 shows an example of a delineated aorta ROI. SUR of the lesions was computed as the ratio of lesion SUV and blood SUV.

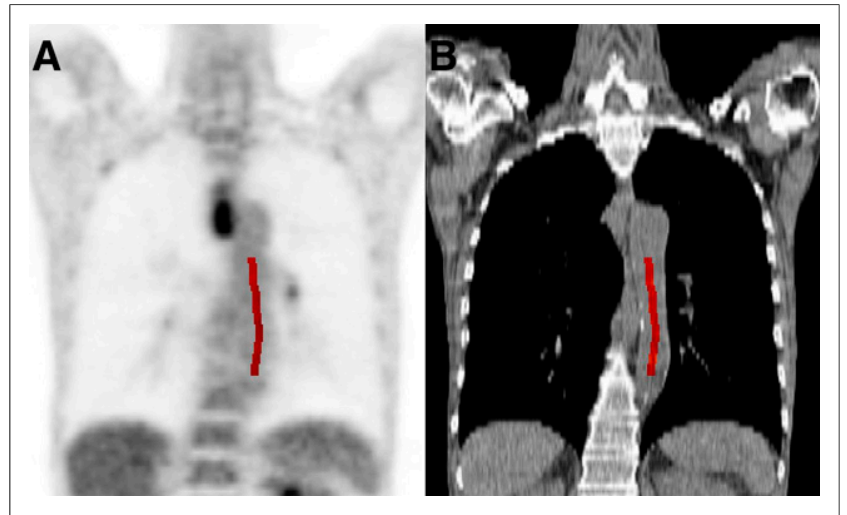

FIGURE 1. Illustration of delineation of aorta. Actual delineation was performed on attenuation $\mathrm{CT}$ ( $\mathrm{B}$, marked in red). Resulting $\mathrm{ROI}$ was then transferred to PET image (A). High tracer uptake of lesion close to aorta will cause spillover into neighboring aorta, which may adversely affect blood SUV determination. Therefore, this part of aorta is excluded from $\mathrm{ROI}$ delineation.
TABLE 2

Summary of Investigated PET Parameters

\begin{tabular}{|c|c|c|}
\hline Parameter & Mean \pm SD & Range \\
\hline MTV (mL) & $21.5 \pm 26.8$ & $1.32-162$ \\
\hline \multicolumn{3}{|l|}{ TLG (mL) } \\
\hline TLG & $218 \pm 299$ & $6.4-1,610$ \\
\hline TLG $^{\text {tc }}$ & $197 \pm 281$ & $6.14-1,500$ \\
\hline TLG & $132 \pm 177$ & $3.82-910$ \\
\hline$T L G_{S U R}^{t c}$ & $112 \pm 159$ & $3.28-809$ \\
\hline \multicolumn{3}{|c|}{ Maximum tracer uptake } \\
\hline $\mathrm{SUV}_{\max }$ & $14.2 \pm 7.2$ & $2.09-34.9$ \\
\hline$S U V_{\max }^{t c}$ & $12.4 \pm 6.43$ & $1.98-34.6$ \\
\hline SUR $_{\max }$ & $8.69 \pm 4.53$ & $1.53-23.2$ \\
\hline$S U R_{\max }^{\text {tc }}$ & $7.06 \pm 3.69$ & $1.28-21.2$ \\
\hline \multicolumn{3}{|c|}{ Mean tracer uptake } \\
\hline $\mathrm{SUV}_{\text {mean }}$ & $8.8 \pm 4.03$ & $1.88-21.8$ \\
\hline$S U V_{\text {mean }}^{\text {tc }}$ & $7.78 \pm 3.61$ & $1.8-21.7$ \\
\hline $\mathrm{SUR}_{\text {mean }}$ & $5.41 \pm 2.54$ & $1.38-13.4$ \\
\hline$S U R_{\text {mean }}^{\text {tc }}$ & $4.43 \pm 2.1$ & $1.16-13.3$ \\
\hline
\end{tabular}

Additionally, lesion SUV and SUR values were scan-time-corrected as described van den Hoff et al. (14):

$$
\begin{gathered}
\mathrm{SUR}^{\mathrm{tc}}=\frac{\mathrm{T}_{0}}{\mathrm{~T}} \times\left(\mathrm{SUR}-\mathrm{V}_{\mathrm{r}}\right)+\mathrm{V}_{\mathrm{r}} \\
\mathrm{SUV}^{\mathrm{tc}}=\mathrm{SUV} \times\left[\frac{\mathrm{SUR}}{\mathrm{SUR}} \times\left(\frac{\mathrm{T}_{0}}{\mathrm{~T}}\right)^{-\mathrm{b}}\right],
\end{gathered}
$$

where $T$ is the actual scan time after injection, and $T_{0}$ is the chosen standard scan time to which the SUV and SUR values are normalized (60 $\mathrm{min}$ in the present work). $\mathrm{V}_{\mathrm{r}}$ is a fixed estimate of the apparent volume of distribution, corresponding to the $y$-axis intercept of a Patlak plot in dynamic investigations (we used $\mathrm{V}_{\mathrm{r}}=0.53 \mathrm{~mL} / \mathrm{mL}$ ), and $\mathrm{b}$ is a parameter describing the shape and decrease of the AIF over time (we used $\mathrm{b}=0.313$ (14)). Corresponding to the 4 possibilities to quantitatively assess the tracer uptake (conventional SUV; scantime-corrected SUV, SUV ${ }^{\text {tc}}$; tumor-to-blood uptake ratio SUR; scantime-corrected tumor-to-blood SUR, SUR ${ }^{\mathrm{tc}}$ ), 4 different measures of maximum tracer uptake, mean tracer uptake, and TLG, respectively, were computed and further analyzed ([Table 2]; subscripts mean and max refer to the mean and maximum value of the respective parameter in the lesion ROI; superscript tc indicates that scan-time correction of SUV and SUR was performed during computation of the respective parameter; and for TLG, the subscript SUR indicates that SUR instead of SUV was used for computation of the TLG product).

\section{Clinical Endpoints and Statistical Analysis}

The 3 clinical endpoints of this study were OS, locoregional tumor control (LRC), and distant metastases-free survival (DM) measured from the start of radiotherapy to death or event. Patients who did not keep follow-up appointments and for whom information on survival or tumor status was thus unavailable were censored with the date of last follow-up.

The association of OS, LRC, and DM with clinically relevant parameters (sex, age, smoking state, histology, grading, T stage, and $\mathrm{N}$ stage) as well as quantitative PET parameters was analyzed using univariate Cox proportional hazards regression in which the PET 
parameters were included as binarized parameters. The cutoffs used for binarization were calculated by performing an univariate Cox regression for each measured value. The value leading to the hazard ratio (HR) with the highest significance was used as cutoff. To avoid too-small group sizes, only values within the interquartile range were considered as potential cutoff. The cutoff values were separately computed for OS, LRC, and DM. The probability of survival was computed and rendered as Kaplan-Meier curves.

Independence of PET parameters from clinically relevant parameters was analyzed in multivariate Cox regression. Those clinical parameters with at least a trend for significance according to univariate Cox regression $(P<0.1)$ were included.

Correlation was tested by the Spearman rank correlation method. Statistical significance was assumed at a $P$ value of less than 0.05 . Statistical analysis was performed with the R Language and Environment for Statistical Computing (version 3.1.2; R Foundation for Statistical Computing) (16).

\section{RESULTS}

The 2-, 3-, and 5-y OS rates were 39\%, 31\%, and 16\%, respectively. These values are in line with data from current literature
(17). Overall, $72 \%$ of patients died during the observation period (last follow-up, October 2014). The median OS was $13 \mathrm{mo}$. In our study, LRC was $46 \%$, and freedom from distant metastases was $34 \%$ among the survivors at $5 \mathrm{y}$.

The values of the investigated PET parameters are summarized in Table 2. Mean blood SUV was $1.7 \% \pm 19 \%$, and the scan-timecorrection factor of lesion SUV was $0.89 \% \pm 11 \%$ on average. Correlation analysis revealed, as expected, a strong correlation of MTV with the different TLG parameters (Spearman $\rho, 0.94-0.95$ ). All TLG parameters and all tracer uptake-based parameters were strongly correlated as well (Spearman $\rho, 0.98-1$ and $0.81-0.98$, respectively). Correlation of MTV and uptake parameters ranged from 0.44 to 0.6 , and correlation of TLG and uptake parameters ranged from 0.62 to 0.79 . All correlations were significant.

\section{os}

OS was significantly associated with all PET parameters with the exception of SUV tc tean. The HRs ranged from 1.6 to 2.5. HRs greater than 2 were found for MTV, SUR $\mathrm{max}_{\text {max }}, \mathrm{SUV}_{\text {mean }}^{\mathrm{tc}}, \mathrm{SUR}_{\text {mean }}$, and SUR tc mean (Table 3). Kaplan-Meier curves with respect to OS are shown in Figure 2.

TABLE 3

Univariate Cox Regression with Respect to OS and DM

\begin{tabular}{|c|c|c|c|c|c|c|c|c|}
\hline \multirow[b]{2}{*}{ Parameter } & \multicolumn{4}{|c|}{ OS } & \multicolumn{4}{|c|}{ DM } \\
\hline & Risk & $\mathrm{HR}$ & $\begin{array}{c}\text { Confidence } \\
\text { interval }\end{array}$ & $P$ & Risk & $\mathrm{HR}$ & $\begin{array}{c}\text { Confidence } \\
\text { interval }\end{array}$ & $P$ \\
\hline \multicolumn{9}{|c|}{ Clinical parameters } \\
\hline Sex & Male & 1.05 & $0.56-1.97$ & 0.88 & Male & 4.53 & $0.62-33.27$ & 0.14 \\
\hline Age & $>61(y)$ & 1.15 & $0.75-1.76$ & 0.51 & $>61(y)$ & 1.43 & $0.66-3.09$ & 0.36 \\
\hline T stage & $>2$ & 1.7 & $1.06-2.71$ & 0.026 & $>2$ & 1.61 & $0.71-3.64$ & 0.25 \\
\hline $\mathrm{N}$ stage & $>0$ & 1.71 & $1.11-2.65$ & 0.016 & $>0$ & 1.31 & $0.62-2.77$ & 0.49 \\
\hline UICC stage & $>I I$ & 1.61 & $1.06-2.46$ & 0.027 & $>I I$ & 1.53 & $0.72-3.26$ & 0.27 \\
\hline Grading & $>2$ & 1.02 & $0.65-1.6$ & 0.92 & $>2$ & 1.57 & $0.71-3.44$ & 0.26 \\
\hline Histology & $\begin{array}{l}\text { Squamous cell } \\
\text { carcinoma }\end{array}$ & 1 & $0.58-1.72$ & 1 & $\begin{array}{l}\text { Squamous cell } \\
\text { carcinoma }\end{array}$ & 0.93 & $0.35-2.44$ & 0.88 \\
\hline Smoker & Yes & 1.85 & $1.12-3.05$ & 0.016 & Yes & 3.35 & $1.15-9.75$ & 0.026 \\
\hline MTV & $>8.5$ & 2.04 & $1.32-3.14$ & 0.001 & $>7.43$ & 2.34 & $1.03-5.33$ & 0.042 \\
\hline \multicolumn{9}{|l|}{ TLG (mL) } \\
\hline TLG & $>124$ & 1.73 & $1.15-2.6$ & 0.0085 & $>47.1$ & 1.95 & $0.86-4.41$ & 0.11 \\
\hline TLG & $>143$ & 1.68 & $1.11-2.56$ & 0.015 & $>41.8$ & 1.95 & $0.86-4.41$ & 0.11 \\
\hline TLG $_{\text {SUR }}$ & $>75.5$ & 1.88 & $1.25-2.82$ & 0.003 & $>75.5$ & 2.12 & $1.02-4.43$ & 0.045 \\
\hline$T_{L G}^{\text {tc }}$ & $>29.1$ & 1.77 & $1.15-2.73$ & 0.0091 & $>38.1$ & 2.08 & $0.96-4.5$ & 0.063 \\
\hline \multicolumn{9}{|l|}{$\begin{array}{l}\text { Maximum tracer } \\
\text { uptake }\end{array}$} \\
\hline$S U V_{\max }$ & $>12.8$ & 1.78 & $1.18-2.69$ & 0.0056 & $>13.6$ & 2.22 & $1.06-4.65$ & 0.035 \\
\hline$S U V_{\max }^{t c}$ & $>10.5$ & 1.57 & $1.02-2.4$ & 0.039 & $>10.5$ & 2.38 & $1.05-5.39$ & 0.037 \\
\hline SUR $_{\max }$ & $>6.17$ & 2.48 & $1.56-3.94$ & $<0.001$ & $>10.2$ & 3.06 & $1.46-6.4$ & 0.003 \\
\hline $\mathrm{SUR}_{\max }^{\mathrm{tc}}$ & $>5.56$ & 2.18 & $1.4-3.37$ & $<0.001$ & $>5.26$ & 4.07 & $1.55-10.71$ & 0.004 \\
\hline \multicolumn{9}{|c|}{ Mean tracer uptake } \\
\hline$S U V_{\text {mean }}$ & $>8.14$ & 1.68 & $1.12-2.52$ & 0.013 & $>6.86$ & 3.29 & $1.25-8.64$ & 0.016 \\
\hline$S U V_{\text {mean }}^{t c}$ & $>6.09$ & 1.4 & $0.9-2.16$ & 0.13 & $>6.09$ & 2.5 & $1.02-6.14$ & 0.046 \\
\hline$S U R_{\text {mean }}$ & $>4.46$ & 2.27 & $1.46-3.53$ & $<0.001$ & $>4.9$ & 4.05 & $1.72-9.52$ & 0.001 \\
\hline$S U R_{\text {mean }}^{\text {tc }}$ & $>3.52$ & 2.11 & $1.34-3.33$ & 0.001 & $>3.75$ & 4.49 & $1.71-11.79$ & 0.002 \\
\hline
\end{tabular}




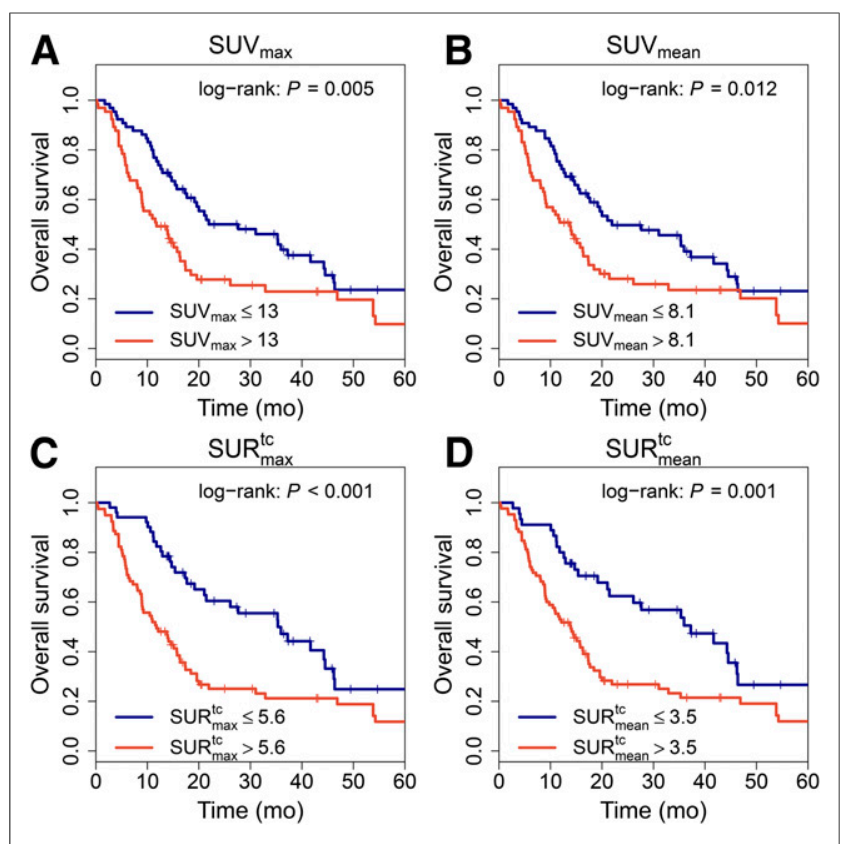

FIGURE 2. Kaplan-Meier curves with respect to OS.

The clinical parameters $\mathrm{T}$ stage, $\mathrm{N}$ stage, UICC stage, and smoking state were significant prognostic factors for OS according to univariate Cox regression $(\mathrm{HR}=1.7, P=0.026 ; \mathrm{HR}=1.71$, $P=0.016 ; \mathrm{HR}=1.61, P=0.027$; and $\mathrm{HR}=1.85, P=0.016$, respectively). These 4 parameters and the PET parameters were included in the multivariate Cox regression. Only MTV, SUR $\mathrm{max}_{\text {, }}$ $\mathrm{SUR}_{\max }^{\mathrm{tc}}, \mathrm{SUR}^{\mathrm{tc}}$, and SUR $\mathrm{R}_{\text {mean }}$ were multivariate significant prognostic factors for OS with similar effect size. TLG, TLG $G_{S U R}$, $\mathrm{TLG}_{\mathrm{SUR}}^{\mathrm{tc}}, \mathrm{SUV}_{\mathrm{max}}$, andSUR $\mathrm{R}_{\text {mean }}^{\text {tc }}$ showed a trend for significance (Table 4). The $P$ value for all other investigated PET parameters was larger than 0.1 .

\section{DM}

For TLG, TLG ${ }^{\text {tc }}$, and TLG $\mathrm{SUR}_{\mathrm{SU}}^{\mathrm{tc}}$, no significant effect was found. All other PET parameters were significant prognostic factors for DM, with HRs ranging from 2.1 to 4.5 (Table 3). The highest HRs were found for $\mathrm{SUR}_{\text {max }}^{\mathrm{tc}}(\mathrm{HR}=4.1)$ and $\mathrm{SUR}_{\text {mean }}^{\text {tc }}(\mathrm{HR}=4.5)$. For $\mathrm{SUV}_{\text {max }}$ and $\mathrm{SUV}_{\text {mean }}$, the HRs were distinctly lower $(\mathrm{HR}=2.2$ and 3.3, respectively). Kaplan-Meier curves with respect to DM are shown in Figure 3.

Among the investigated clinical parameters only smoking state was prognostic for $\mathrm{DM}$ in univariate $(\mathrm{HR}=3.35, P=0.026)$ and multivariate analysis $(\mathrm{HR}=3.1, P=0.04)$. The results of multivariate Cox regression including this parameter and the PET parameters are shown in Table 5. MTV- and all SUR-based parameters were prognostic for DM; $\mathrm{SUV}_{\text {max/mean }}$ showed a trend for significance. The $P$ value for all other investigated PET parameters was larger than 0.1. The highest HRs were found for $S_{U R} R_{\max }^{\text {tc }}$ $(\mathrm{HR}=3.9, P=0.014)$ and $\mathrm{SUR}_{\text {mean }}^{\text {tc }}(\mathrm{HR}=4.4, P=0.007)$.

\section{LRC}

Univariate Cox regression with respect to LRC revealed a significant effect only for $\mathrm{N}$ stage greater than $0(P=0.048)$. For patient age higher than $61 \mathrm{y}$, a trend for significance was found $(P=$ 0.052). The $P$ value for all other investigated parameters was larger than 0.1 . Therefore, this clinical endpoint was not further analyzed.

\section{DISCUSSION}

In this exploratory study, we investigated the prognostic value of pretherapeutic SUR (with and without scan-time correction) and scan-time-corrected SUV in comparison to MTV, TLG, and SUV under consideration of known clinical prognostic parameters in patients with locally advanced esophageal carcinoma treated with definitive chemoradiotherapy.

Our main result was that scan-time correction of lesion SUV and, more pronouncedly, normalization of (scan-time-corrected) lesion SUV to blood SUV (i.e., the use of SUR instead of SUV) increased the prognostic value of the thus quantified tracer uptake that served as a surrogate of the lesion's glucose consumption. A second result was that ${ }^{18} \mathrm{~F}$-FDG $\mathrm{PET}$ parameters in general provide independent prognostic information for OS and DM but not for LRC in this patient group. The only clinical parameter remaining significant in multivariate analysis performed together with the PET parameters was smoking status of the patients.

For OS, multivariate analyses revealed significant correlations only for MTV, SUR $\mathrm{max}_{\max }, \mathrm{SUR}_{\max }^{\text {tc }}$, and $\mathrm{SUR}_{\text {mean }}$ TLG and $\mathrm{SUV}_{\text {max/mean }}$ did not show a significant effect. This result is in agreement with a recent publication by Lemarignier et al. (8) in which MTV was prognostic for OS, whereas $\mathrm{SUV}_{\max }$ was not prognostic. Normalization to blood SUV and scan-time correction of SUV improved the prognostic value in our patient group, leading to comparable HRs and levels of significance for MTV and SUR values. For DM, $\mathrm{SUV}_{\max }$ and $\mathrm{SUV}_{\text {mean }}$ were significant predictors according to multivariate Cox regression. However, the HR of scan-time-corrected SUR was notably larger than that of MTV or SUV-for example, the HR of SUR $\mathrm{max}_{\max }^{\mathrm{tc}}$ with respect to DM was 3.9, compared with 2.2, for $\mathrm{SUV}_{\max }$ - suggesting that SUR may have more potential

TABLE 4

Multivariate Cox Regression with Respect to OS

\begin{tabular}{llll}
\hline \multicolumn{1}{c}{ Parameter } & HR & Confidence interval & $P$ \\
\hline Clinical parameters & & & \\
\hline T stage & 1.5 & $0.75-2.8$ & 0.27 \\
\hline N stage & 1.3 & $0.71-2.5$ & 0.38 \\
\hline UICC stage & 0.84 & $0.41-1.7$ & 0.64 \\
\hline Smoker & 1.7 & $1-2.8$ & 0.045 \\
\hline PET parameters & & & \\
\hline MTV & 1.8 & $1.1-2.9$ & 0.019 \\
\hline TLG & 1.5 & $0.94-2.4$ & 0.086 \\
\hline TLG $_{\text {suR }}^{\text {tc }}$ & 1.6 & $0.95-2.7$ & 0.083 \\
\hline TLG $_{\text {SUR }}$ & 1.6 & $0.94-2.8$ & 0.084 \\
SUV $_{\text {max }}$ & 1.6 & $0.99-2.7$ & 0.056 \\
\hline SUR $_{\text {max }}$ & 2 & $1.2-3.6$ & 0.012 \\
\hline SUR $_{\text {max }}^{c}$ & 1.9 & $1.1-3.2$ & 0.019 \\
\hline SUR $_{\text {mean }}$ & 2.1 & $1.2-3.7$ & 0.01 \\
\hline SUR $_{\text {mean }}^{\text {c }}$ & 1.7 & $0.95-3.1$ & 0.071 \\
\hline
\end{tabular}

Each PET parameter was analyzed separately together with clinical parameters, which were significant prognostic factors (or exhibited a trend for significance) in univariate Cox regression. HRs and $P$ values of clinical parameters were averaged over all analyses. 


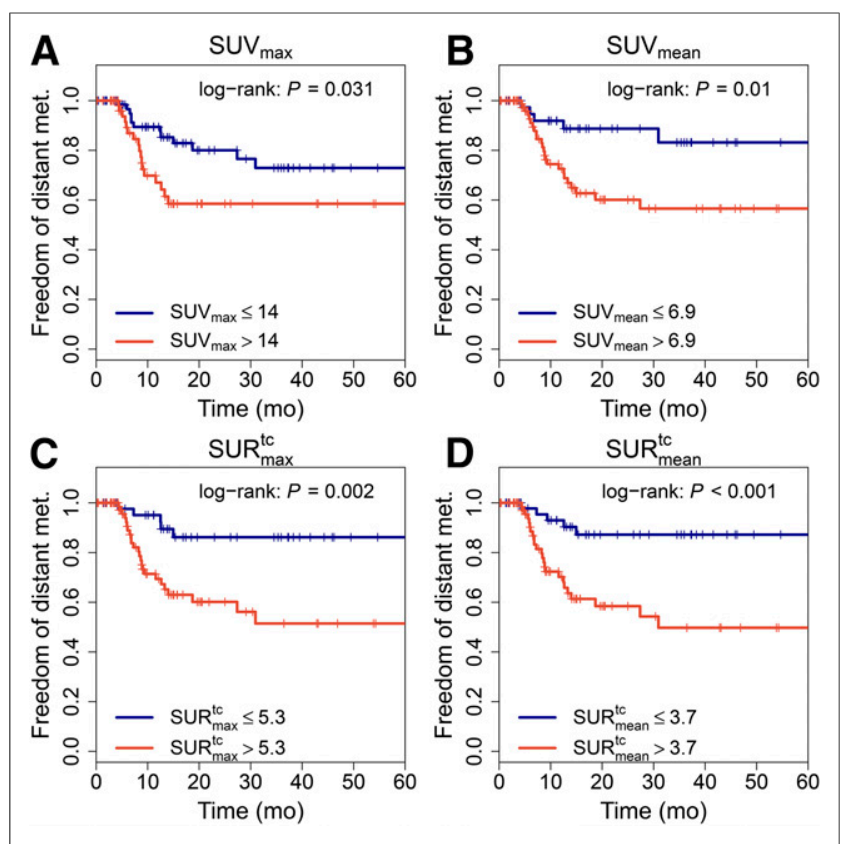

FIGURE 3. Kaplan-Meier curves with respect to DM.

than the other PET parameters to predict occurrence of DM after therapy.

The largest HR with respect to DM was found for SUR tc thean, the scan-time-corrected mean SUR (Table 5). Surprisingly, this did not lead to a prognostic value of TLG tc SUR, which was not significantly correlated with DM. A plausible explanation would be that the prognostic value of TLG is dominated by MTV (strongly suggested by the pronounced linear correlation between TLG and MTV) whereas the influence of the tracer uptake on variations of TLG is less pronounced.

As our results demonstrate, both MTV and SUR provide prognostic information for OS and development of distant metastases that add to clinical parameters. However, MTV in the present study was determined using an essentially fully automated delineation algorithm. Although several viable automated algorithms have been published (18-27), in many institutions MTV is presently still determined by manual delineation. Manual delineation is known to be prone to intraand interobserver variability as well as to potentially gross size and background-dependent bias if fixed absolute or relative thresholds are used. Therefore, the prognostic value of MTV may potentially be less convincing when the lesions are delineated manually. In contrast, $\mathrm{SUR}_{\max }^{\mathrm{tc}}$ and $\mathrm{SUV}_{\max }$ have the clear advantage that they are independent of the details of the delineation and can always be determined unambiguously.

In our study, scan-time correction of SUV did generally not improve the prognostic value of SUV. This finding indicates that in our patient group the improved prognostic value of SUR is caused mainly by the beneficial influence of normalization to blood SUV rather than by scan-time correction because the variability of the blood SUV (19\%) is larger than that of the scan-time correction factor (11\%).

Another possible explanation is that scan-time correction of SUV requires stronger assumptions than that of SUR. In the latter case, it has only to be assumed that the AIF can be described by some power law starting early after bolus passage, which empirically is fulfilled to a good degree (14). For scan-time correction of SUV it is necessary to assume that all AIFs can be described with the same power law-that is, the exponent $\mathrm{b}$ in Equation 1 is identical for all AIFs and its value directly enters the correction formula Equation 1. Any interindividual residual variability of $b$ would adversely affect the accuracy of the SUV scan-time correction and might thus explain the failure to improve the prognostic value of the SUV. Further investigations will be necessary to settle this question.

Regarding the superior performance of SUR in the present study, it should be emphasized that in comparison to SUV its determination additionally requires the thorough delineation of an aorta ROI to derive the blood SUV. Principally, this introduces a further potential source of error including intra- and interobserver variability effects. But fortunately, the aorta (and its boundaries) can be quite easily and unambiguously identified in the CT data, and it is also easy to observe the safety margin (and to possibly exclude aorta sections affected by high tracer uptake in immediately adjacent structures), as illustrated in Figure 1, to obtain unbiased blood SUV values. Overall, if the necessary care is taken during the aorta delineation the reproducibility of the blood SUV (and reproducibility of the resulting SUR) is high.

The fact that only $\mathrm{N}$ stage as a clinical parameter and none of the PET parameters in our patient cohort was significantly correlated with LRC is somewhat surprising. However, in contrast to overall and disease-free survival local control after primary radiochemotherapy of esophageal cancer is scarcely reported in the literature, and we are aware of only 2 studies correlating this endpoint with PET-derived parameters $(6,7)$. Most of our patients had T3 and N1 tumors and represent, therefore, a comparably narrow range of tumor burden undergoing homogeneous treatment, making it difficult to identify prognostic factors in the sample size reported here. Statistical power is further diminished by the fact that the LRC rate in our study was $46 \%$ after $5 \mathrm{y}$-that is, the number of events for this endpoint was considerably less than the number of events for distant metastases or death. In addition, almost all of the patients were deemed as having unresectable tumors or unfit for surgery by the multidisciplinary cancer team so that life expectancy may be affected not only by distant metastases but also by comorbidity. Both of these confounders

TABLE 5

Multivariate Cox Regression with Respect to DM

\begin{tabular}{llll}
\hline \multicolumn{1}{c}{ Parameter } & HR & Confidence & $P$ \\
\hline $\begin{array}{l}\text { Clinical parameters } \\
\text { Smoker }\end{array}$ & 1.7 & $0.95-3.1$ & 0.071 \\
\hline $\begin{array}{l}\text { PET parameters } \\
\text { MTV }\end{array}$ & 2.5 & $1-6$ & 0.039 \\
\hline SUV & & & \\
SUR $_{\text {max }}$ & 2.2 & $0.99-4.7$ & 0.052 \\
SUR $_{\text {max }}^{\text {cc }}$ & 3.1 & $1.4-6.7$ & 0.005 \\
SUV $_{\text {mean }}$ & 3.9 & $1.3-11$ & 0.014 \\
SUR $_{\text {mean }}$ & 2.5 & $0.94-6.7$ & 0.065 \\
SUR $_{\text {mean }}^{\text {c }}$ & 3.3 & $1.4-7.9$ & 0.007 \\
\hline & 4.4 & $1.5-13$ & 0.007 \\
\hline
\end{tabular}

Each PET parameter was analyzed separately together with clinical parameters, which were significant prognostic factors (or exhibited a trend for significance) in univariate Cox regression. HRs and $P$ values of clinical parameters were averaged over all analyses. 
may have interfered with detection of recurrent tumors after radical radiochemotherapy in our study.

A general limitation of our study was its retrospective explorative character - that is, our findings have to be considered as hypothesis-generating and preliminary. Therefore, our results need validation in further studies with independent patient groups before final conclusions on the prognostic value of the described parameters can be drawn. In the case that the prognostic value will be confirmed, the predictive value of the described PET parameters needs to be addressed in prospective stratification or intervention studies.

\section{CONCLUSION}

PET provides independent prognostic information for OS and DM but not for LRC in patients with locally advanced esophageal carcinoma treated with definitive radiochemotherapy in addition to clinical parameters. Among the investigated uptake-based parameters, only SUR was an independent prognostic factor for OS and DM. These results suggest that the prognostic value of tracer uptake can be improved when characterized by SUR instead of SUV. Further investigations are required to confirm these preliminary hypothesis-generating results.

\section{DISCLOSURE}

The costs of publication of this article were defrayed in part by the payment of page charges. Therefore, and solely to indicate this fact, this article is hereby marked "advertisement" in accordance with 18 USC section 1734. This work was supported in part by the German Federal Ministry of Education and Research (BMBF contract 03ZIK041). No other potential conflict of interest relevant to this article was reported.

\section{REFERENCES}

1. van Hagen P, Hulshof M, Van Lanschot J, et al. Preoperative chemoradiotherapy for esophageal or junctional cancer. N Engl J Med. 2012;366:2074-2084.

2. Zhang Y. Epidemiology of esophageal cancer. World J Gastroenterol. 2013;19: 5598-5606.

3. Omloo JM, van Heijl M, Hoekstra O, van Berge Henegouwen M, Van Lanschot J, Sloof G. FDGPET parameters as prognostic factor in esophageal cancer patients: a review. Ann Surg Oncol. 2011;18:3338-3352.

4. Van de Wiele C, Kruse V, Smeets P, Sathekge M, Maes A. Predictive and prognostic value of metabolic tumour volume and total lesion glycolysis in solid tumours. Eur J Nucl Med Mol Imaging. 2013;40:290-301.

5. Suzuki A, Xiao L, Hayashi Y, et al. Prognostic significance of baseline positron emission tomography and importance of clinical complete response in patients with esophageal or gastroesophageal junction cancer treated with definitive chemoradiotherapy. Cancer. 2011;117:4823-4833.

6. Atsumi K, Nakamura K, Abe K, et al. Prediction of outcome with FDG-PET in definitive chemoradiotherapy for esophageal cancer. J Radiat Res (Tokyo). 2013; 54:890-898.
7. Amini A, Ajani J, Komaki R, et al. Factors associated with local-regional failure after definitive chemoradiation for locally advanced esophageal cancer. Ann Surg Oncol. 2014;21:306-314.

8. Lemarignier C, Di Fiore F, Marre C, et al. Pretreatment metabolic tumour volume is predictive of disease-free survival and overall survival in patients with oesophageal squamous cell carcinoma. Eur J Nucl Med Mol Imaging. 2014;41: 2008-2016.

9. Hamberg LM, Hunter G, Alpert N, Choi N, Babich J, Fischman A. The dose uptake ratio as an index of glucose metabolism: useful parameter or oversimplification? J Nucl Med. 1994;35:1308-1312.

10. Keyes JW Jr. SUV: standard uptake or silly useless value? J Nucl Med. 1995; 36:1836-1839.

11. Huang SC. Anatomy of SUV. Nucl Med Biol. 2000;27:643-646.

12. Boktor RR, Walker G, Stacey R, Gledhill S, Pitman AG. Reference range for intrapatient variability in blood-pool and liver SUV for ${ }^{18}$ F-FDG PET. $J$ Nucl Med. 2013;54:677-682.

13. van den Hoff J, Oehme L, Schramm G, et al. The PET-derived tumor-to-blood standard uptake ratio (SUR) is superior to tumor SUV as a surrogate parameter of the metabolic rate of FDG. EJNMMI Res. 2013;3:77.

14. van den Hoff J, Lougovski A, Schramm G, et al. Correction of scan time dependence of standard uptake values in oncological PET. EJNMMI Res. 2014;4:18.

15. Hofheinz F, Langner J, Petr J, et al. An automatic method for accurate volume delineation of heterogeneous tumors in PET. Med Phys. 2013;40:082503108250310 .

16. R Core Team. R: A Language and Environment for Statistical Computing. Vienna, Austria: R Foundation for Statistical Computing; 2014.

17. Cooper JS, Guo MD, Herskovic A, et al. Chemoradiotherapy of locally advanced esophageal cancer: long-term follow-up of a prospective randomized trial (RTOG 85-01). JAMA. 1999;281:1623-1627.

18. Erdi YE, Mawlawi O, Larson S, et al. Segmentation of lung lesion volume by adaptive positron emission tomography image thresholding. Cancer. 1997;80:2505-2509.

19. Daisne JF, Sibomana M, Bol A, Doumont T, Lonneux M, Gregoire V. Tridimensional automatic segmentation of PET volumes based on measured sourceto-background ratios: influence of reconstruction algorithms. Radiother Oncol. 2003;69:247-250.

20. Boellaard R, Krak N, Hoekstra O, Lammertsma A. Effects of noise, image resolution, and ROI definition on the accuracy of standard uptake values: a simulation study. J Nucl Med. 2004;45:1519-1527.

21. Black QC, Grills I, Kestin L, et al. Defining a radiotherapy target with positron emission tomography. Int J Radiat Oncol Biol Phys. 2004;60:1272-1282.

22. Nestle U, Kremp S, Schaefer-Schuler A, et al. Comparison of different methods for delineation of 18 F-FDG PET-positive tissue for target volume definition in radiotherapy of patients with non-small cell lung cancer. $J$ Nucl Med. 2005;46: 1342-1348.

23. Drever L, Robinson D, McEwan A, Roa W. A local contrast based approach to threshold segmentation for PET target volume delineation. Med Phys. 2006; 33:1583-1594.

24. van Dalen JA, Hoffmann A, Dicken V, et al. A novel iterative method for lesion delineation and volumetric quantification with FDG PET. Nucl Med Commun. 2007;28:485-493.

25. Jentzen W, Freudenberg L, Eising E, Heinze M, Brandau W, Bockisch A. Segmentation of PET volumes by iterative image thresholding. J Nucl Med. 2007;48:108-114.

26. Vauclin S, Doyeux K, Hapdey S, Edet-Sanson A, Vera P, Gardin I. Development of a generic thresholding algorithm for the delineation of ${ }^{18}$ FDG-PET-positive tissue: application to the comparison of three thresholding models. Phys Med Biol. 2009;54:6901-6916.

27. Frings V, de Langen A, Smit E, et al. Repeatability of metabolically active volume measurements with ${ }^{18} \mathrm{~F}-\mathrm{FDG}$ and ${ }^{18} \mathrm{~F}$-FLT PET in non-small cell lung cancer. J Nucl Med. 2010;51:1870-1877. 